\title{
A propos de la validité d' Icbtbyocotylurus variegatus (Creplin, 1825) et de la position spécifique de Tetracotyle ovata (v. Linstow, 1877) (Trematoda : Strigeidae)
}

\author{
par K. ODENING \\ Forschungsstelle für Wirbeltierforschung (im Tierpark Berlin) \\ der Akademie der Wissenschaften der DDR, 1136 Berlin, Am Tierpark 125.
}

\section{Résumé.}

Ichthyocotylurus variegatus (Creplin, 1825) est une espèce indépendante, distincte d'Ichthyocotylurus platycephalus (Creplin, 1825). On souligne les différences morphologiques observées entre les métacercaires de ces deux espèces (Tetracotyle percaefluviatilis v. Linstow, $1877=I$. variegatus et Tetracotyle ovata v. Linstow, $1877=$ « $T$. variegata sensu Hughes, $1928=\ll T$. pileata $»$ sensu Dubois, $1938,1968=T$. communis Hughes, $1928=I$. platycephalus) et la localisation précise et différente des adultes (maritae) dans les Oiseaux hôtes définitifs (I. variegatus dans le tiers postérieur de l'intestin grêle, I. platycephalus dans la région du cloaque). On donne une diagnose et la synonymie des métacercaires des quatre espèces d'Ichthyocotylurus et on propose une clé de détermination.

\section{Summary.}

On the validity of Ichthyocotylurus variegatus (Creplin, 1825) and the species affiliation of Tetracotyle ovata (v. Linstow, 1877) (Trematoda : Strigeidae).

Ichthyocotylurus variegatus (Creplin, 1825) is a species of its own, differing from Ichthyocotylurus platycephalus (Creplin, 1825). The distinct morphological difference between the metacercariae of the two species must be stressed (Tetracotyle percaefluviatilis $\mathrm{v}$. Linstow, $1877=I$. variegatus, Tetracotyle ovata v. Linstow, $1877=$ 《 $T$. variegata sensu Hughes, $1928=\ll T$. pileata $»$ sensu Dubois, $1938,1968=T$. communis Hughes, $1928=I$. platycephalus), as well as the different location of the maritae in the definitive host (I. variegatus in the posterior third of the small intestine, I. platycephalus in the region of the cloaca). A key for the determination, synonymes and diagnoses of the metacercariae of the 4 Ichthyocotylurus species are presented.

Accepté le 9 octobre 1978. 
Il y a peu de temps, Dubois (1978) a affirmé, en se basant sur l'étude du matériel original (maritae), l'identité d'Ichthyocotylurus platycephalus (Creplin, 1825) (syn. Holostomum cucullus Thoss, 1897) et de I. variegatus (Creplin, 1825) (syn. Cotylurus cumulitestis Dubois, 1962). L'analyse de ce travail démontre que la différenciation morphologique des adultes (maritae) de ces deux espèces, est actuellement difficile (ce qui n'est pas extraordinaire, car elles correspondent à ce que l'on nomme des espèces jumelles [sibling species]) (1).

Par contre, les adultes des deux espèces se distinguent très nettement par leur localisation dans l'Oiseau, hôte définitif. I. platycephalus se trouve dans le cloaque et/ou la bourse de Fabricius, I. variegatus dans le tiers postérieur de l'intestin grêle (il arrive cependant que l'habitat des deux espèces chevauche au niveau du rectum).

La réalisation expérimentale du passage cercaire-métacercaire des deux espèces a prouvé qu'il existe des différences morphologiques chez ces deux stades larvaires (Odening, Mattheis et Bockhardt, 1970; Odening et Bockhardt, 1971).

Dubois (1978) n'a traité que les différences entre les cercaires, différences qu'il a estimées très faibles et insuffisantes pour séparer les espèces (voir par contre l'exposé de Odening et Bockhardt, 1971).

Cependant Dubois (1978) n'a pas fait mention des différences morphologiques très nettes qui existent entre les métacercaires. A ce sujet, Odening et Bockhardt (1971) ont écrit: «Pour séparer les deux espèces de façon précise, il est indispensable d'examiner au microscope les métacercaires dékystées afin de mesurer les dimensions de la ventouse ventrale et de l'organe tribocytique. La taille de la ventouse ventrale et ses proportions par rapport à l'organe tribocytique constituent des caractères distinctifs fiables. »

La métacercaire d'Ichthyocotylurus variegatus est identique à Tetracotyle percaefluviatilis v. Linstow, 1877 et celle de I. platycephalus à Tetracotyle ovata v. Linstow, 1877 (syn. « $T$. variegata » sensu Hughes, $1928=$ = $T$. pileata » sensu Dubois, 1938, 1968, T. communis Hughes, 1928).

$\mathrm{Vu}$ la confusion qui persiste entre les noms donnés aux métacercaires et ceux attribués aux adultes, nous donnons ci-dessous une brève diagnose et une clé de détermination des métacercaires des quatre espèces d'Ichthyocotylurus, en indiquant les synonymies qui nous paraissent établies.

Les métacercaires des Ichthyocotylurus n'ont pas une forme en gobelet mais elles sont massives. Dékystées, elles sont arrondies, elliptiques ou ovales. La partie postérieure, très petite, ne se différencie pas nettement de la partie antérieure du corps. L'organe tribocytique, situé dans la partie postérieure, n'est pas lobé. La métacercaire est plus ou moins sensible à la pression. Sauf indication contraire, les mesures données constituent une combinaison de celles prises sur le vivant et de celles d'individus montés in toto. Pour les quatre espèces, nous nous sommes servi d'observations personnelles

(1) La différenciation morphologique des adultes de $I$. platycephalus et de $I$. variegatus est possible si on compare statistiquement la forme et les proportions du corps des deux espèces, chez des exemplaires de même largcur (cf. Razmaškin, 1966; Odening, Mattheis et Bockhardt, 1970 ; Odening et Bockhardt, 1971). 
inédites et de celles de Hughes (1928), Razmaškin (1966, 1974, 1976). Odening, Mattheis et Bockhardt (1970), Olson (1970), Niewiadomska et Kozicka (1970) et Odening et Bockhardt (1971). La clé que nous proposons ci-dessous est basée sur celle de Razmaškin (1966) à laquelle nous avons apporté quelques changements.

Il y a contradiction entre les indications de Hughes (1928) et Razmaškin (1966) d'une part et celles de Niewiadomska et Kozicka (1970) d'autre part, concernant la longueur des pseudo-ventouses chez la métacercaire de $I$. erraticus. Il semble que non seulement Hughes mais aussi Razmaškin aient mesuré seulement la cavité des pseudoventouses. C'est pourquoi nous nous fondons ici sur les dimensions des pseudo-ventouses de la métacercaire de $I$. erraticus indiquées sur les dessins de Niewiadomska et Kozicka (1970) et qui correspondent à nos mesures.

\section{Clé de détermination des métacercaires d'Ichthyocotylurus.}

1. Capsule et kyste faciles à déchirer; enveloppe hyaline (parasitogène) mince, enveloppe de la capsule formée par l'hôte (xeniogène) mince ou épaisse (fig. $1 a-b) \ldots \ldots \ldots 2$

- Capsule et kyste difficiles à déchirer ; enveloppe du kyste (parasitogène) et capsule (xenio-

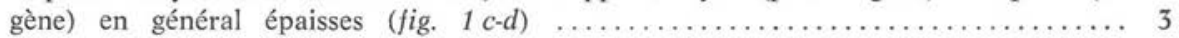

2. Largeur de la ventouse ventrale supérieure à $150 \mu \mathrm{m}$; organe tribocytique (93-161 $\mu \mathrm{m}$ de longueur) plus court ou aussi long (rarement à peine plus long) que la ventouse ventrale (109-257 $\mu \mathrm{m}$ de longueur) ; pseudo-ventouses (52-155 $\mu \mathrm{m}$ de longueur) aussi longues ou légèrement plus longues que la ventouse buccale $(93-150 \mu \mathrm{m}$ de longueur) $\ldots \ldots$. $\ldots \ldots \ldots \ldots \ldots \ldots \ldots \ldots \ldots \ldots \ldots \ldots \ldots \ldots \ldots \ldots \ldots \ldots \ldots \ldots \ldots \ldots \ldots \ldots \ldots \ldots \ldots$ I. platycephalus (fig. $2 a$ )

- Largeur de la ventouse ventrale inférieure à $115 \mu \mathrm{m}$; organe tribocytique $(110-276 \times 132$ $345 \mu \mathrm{m})$ nettement plus long et plus grand que la ventouse ventrale $(62-110 \times 77$ $114 \mu \mathrm{m}) ;$ pseudo-ventouses plus longues $(88-171 \mu \mathrm{m})$ que la ventouse buccale $(65-90 \mu \mathrm{m})$

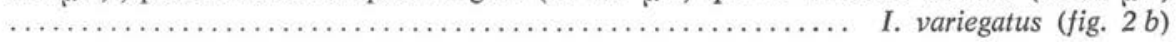

3. Ventouse ventrale $(50-90 \mu \mathrm{m})$ un peu plus large que la ventouse buccale $(40-70 \mu \mathrm{m})$; pseudo-ventouses plus longues que la ventouse buccale ......... I. erraticus (fig. $2 c$ )

- Ventouse ventrale $(35-66 \mu \mathrm{m})$ aussi large que la ventouse buccale ; pseudo-ventouses plus courtes que la ventouse buccale $\ldots \ldots \ldots \ldots \ldots \ldots \ldots \ldots \ldots \ldots \ldots \ldots \ldots \ldots$. pileatus (fig. $2 d$ )

\section{Synonymies et diagnoses des métacercaires.}

$$
\text { Icbtbyocotylurus platycepbalus (Creplin, 1825). }
$$

Synonymes :

Tetracotyle echinata Diesing, 1858 (2).

T. ovata v. Linstow, 1877.

T. communis Hughes, 1928.

T. biwaensis Goto et Ozaki, 1930 (cf. Skrjabin, 1974).

T. tahoensis Haderlie, 1953 (cf. Skrjabin, 1974).

T. leucisci Sidorov, 1956 (cf. Skrjabin, 1974).

(2) Dujardin (1845, p. 463, à la suite du $\mathrm{n}^{\circ}$ 139) a signalé des métacercaires enkystées chez un « gardon» (qui n'était pas Cyprinus idus) qui correspondent à cette espèce. Le travail de Dollfus (1968, p. 159 pl. XXXI : «Tetracotyle cypriniidi»), rend en effet possible la détermination de ces métacercaires. Claparède (1858) a mentionné aussi ce Trématode. 

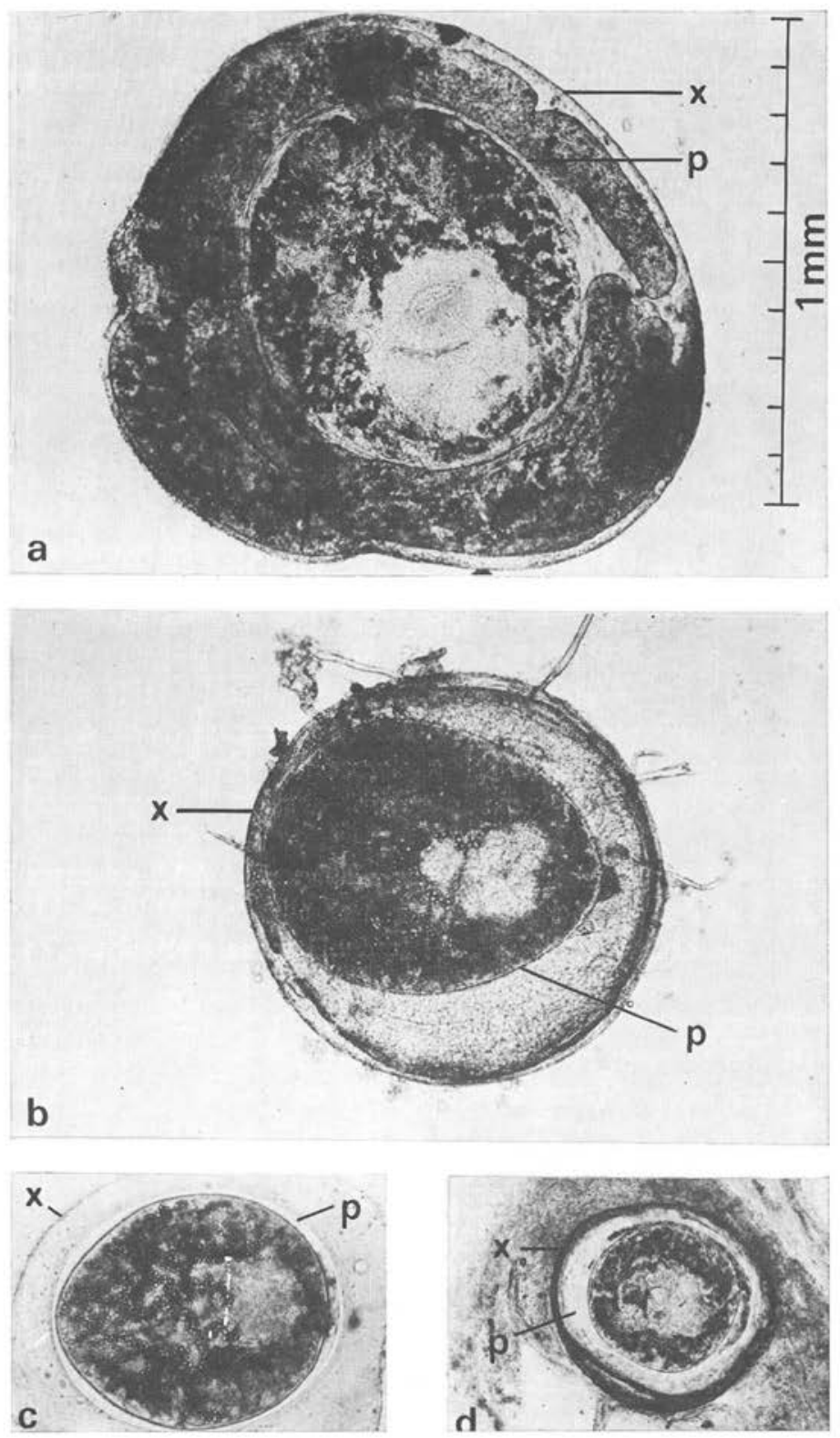

Fig. 1. Capsules (x) et kystes (p) des métacercaires d'Ichthyocotylurus. a) I. plutycephalus de Lucioperca lucioperca; b) I. variegatus de Perca fluviatilis; c) $I$. erraticus d'Osmerus eperlanus; d) $I$. pileatus de Perca fluviatilis (toutes de la R.D.A.). 


\section{Diagnose.}

- Métacercaires dans des capsules globuleuses ou ovoïdes mesurant 536-1 334 $\times 448-980 \mu \mathrm{m}$. Enveloppe du kyste mince, élastique et très sensible à la pression

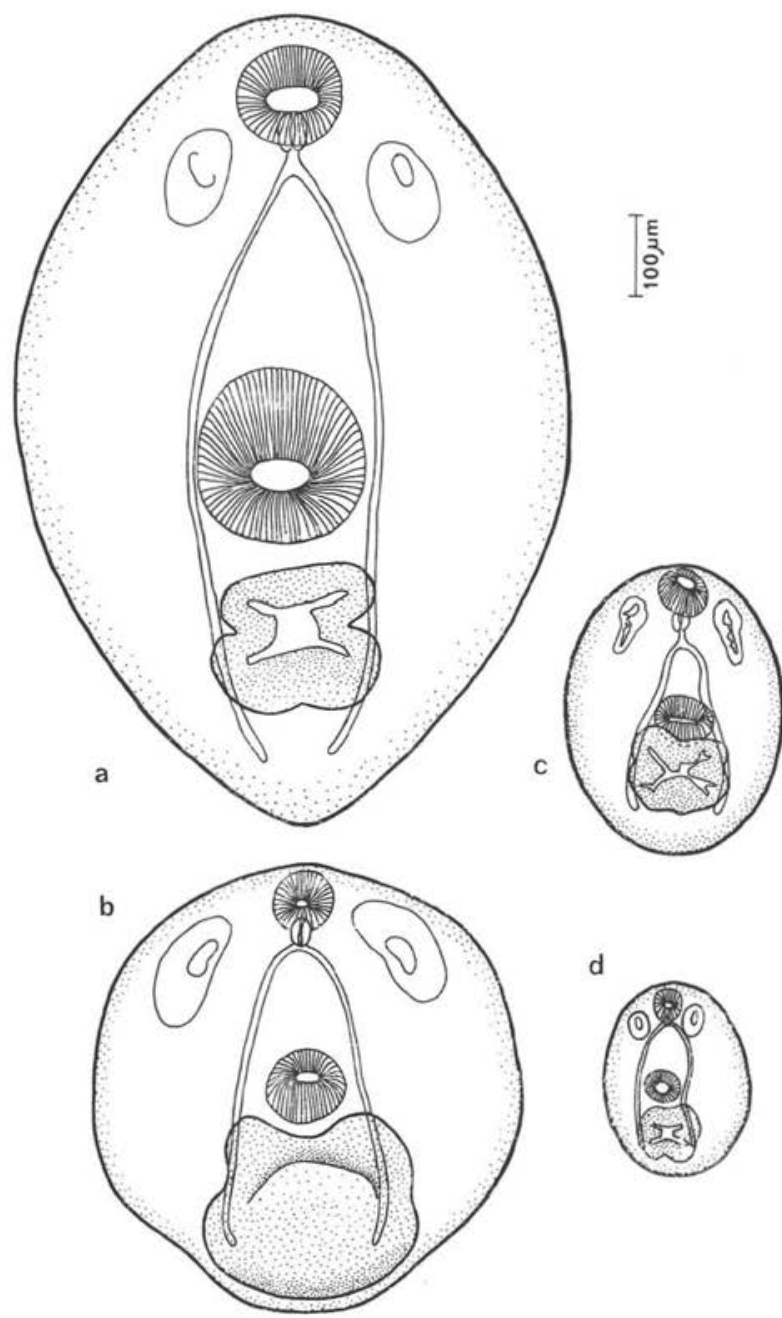

Fig. 2. Métacercaires dékystées d'Ichthyocotylurus. a) I. platycephalus; b) I. variegatus; c) I. erraticus; d) I. pileatus (c d'après Niewiadomska et Kozicka 1970 de la Pologne, les autres de la R.D.A.). 
et par conséquent facile à déchirer. Corps de la métacercaire 510-1 $300 \times 342-900 \mu \mathrm{m}$ (3). Ventouse buccale $87-150 \times 107-155 \mu \mathrm{m}$. Pharynx 35-52 $\times 22-41 \mu \mathrm{m}$. Pseudoventouses $52-155 \times 39-150 \mu \mathrm{m}$. Ventouse ventrale $109-257 \times 150-250 \mu \mathrm{m}$. Organe tribocytique $92-200 \times 140-367 \mu \mathrm{m}$ (fig. 1 a, $2 a$ ).

- A été signalé dans de nombreux Téléostéens d'eau douce appartenant à des groupes systématiques différents (Perciformes, Percopsiformes, Cypriniformes, Salmonidae, Osmeridae, Esocidae, Gadiformes, Gasterosteiformes).

- Localisation préférentielle dans la région du cœur, mais on les trouve aussi dans la cavité générale du corps, plus rarement ailleurs (cavité des yeux, paroi de la vessie gazeuse). Cf. Hughes (1928) ; Šigin (1961, 1966: «T. communis ») ; Razmaškin (1963, 1966: «T. communis», 1974, 1976) ; Odening, Mattheis et Bockhardt (1970).

- Région holarctique.

\section{Icbtbyocotylurus variegatus (Creplin, 1825)}

Synonyme: Tetracotyle percaefluviatilis von Linstow, 1877 (4).

\section{Diagnose.}

- Métacercaires dans des capsules globuleuses ou ovoïdes de $570-800 \times 400$ $752 \mu \mathrm{m}$. Enveloppe du kyste mince, assez sensible à la pression et facile à déchirer. Corps 380-880 $\times$ 300-771 $\mu \mathrm{m}$. Ventouse buccale 65-90 $\times 60-104 \mu \mathrm{m}$. Pharynx 22-35 $\times 17-40 \mu \mathrm{m}$. Pseudoventouses 88-171 $\times$ 30-66 $\mathrm{m}$. Ventouse ventrale $62-110 \times 77$. $114 \mu \mathrm{m}$. Organe tribocytique 110-276 $\times 132-345 \mu \mathrm{m}$ (fig. $1 \mathrm{~b}, 2 \mathrm{~b}$ ).

- Se trouve chez les Percidae, le plus souvent chez Perca fluviatilis, mais on peut aussi la récolter chez Osmerus.

- Elle est en général localisée dans la paroi de la vessie gazeuse, sur le péritoine, le mésentère mais aussi dans la cavité générale du corps y compris la région cardiaque et les reins. Cf. Šigin (1961, 1966: «T. platycephalus ») ; Razmaškin (1963, 1966 : «T. platycephalus », 1974, 1976); Odening et Bockhardt (1971).

- Région paléarctique.

\section{Icbthyocotylurus erraticus (Rudolphi, 1809)}

Synonymes :

Tetracotyle intermedia Hughes, 1928.

T. coregoni Achmerov, 1941.

(3) Un exemplaire figuré par Niewiadomska (1970) a une longueur insolite de $1750 \mu \mathrm{m}$.

(4) Moulinié (1856) a, le premier, décrit cette métacercaire sous le nom de «Tetracotyle de la Perche fluviatile». 


\section{Diagnose.}

- Dans des capsules ovoïdes ou globuleuses mesurant 480-1 $480 \times 400-1110 \mu \mathrm{m}$ (enveloppe xeniogène de 150-510 $\mu \mathrm{m}$ d'épaisseur). Kystes ovoïdes, avec enveloppe épaisse, difficiles à déchirer. Corps 232-590 × 160-600 $\mu \mathrm{m}$. Ventouse buccale 43-75

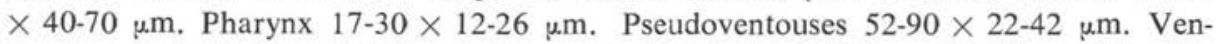
touse ventrale $40-76 \times 50-90 \mu \mathrm{m}$. Organe tribocytique $66-140 \times 90-150 \mu \mathrm{m}$ (fig. $1 \mathrm{c}$, $2 c)$.

- Chez les Salmonidae, Thymallidae, Osmeridae, plus rarement chez les Dalliidae, Cottidae, et Cyprinidae.

- Localisation sur le cœur, les reins, plus rarement dans les gonades et la cavité générale du corps ou les yeux. Cf. Hughes (1928) ; Razmaškin (1963, 1966, 1974, 1976) ; Niewiadomska et Kozicka (1970) ; Olson (1970).

- Région holarctique.

\section{Icbthyocotylurus pileatus (Rudolphi, 1802)}

Synonyme: Tetracotyle diminuta Hughes, 1928.

\section{Diagnose.}

- Dans des capsules globuleuses ou ovö̈des mesurant 285-565 $\times 266-528 \mu \mathrm{m}$. Enveloppe du kyste épaisse, difficile à déchirer. Corps 155-429 × 134-342 $\mu \mathrm{m}$. Ventouse buccale 35-66 $\times 35-66 \mu \mathrm{m}$. Pharynx 13-26 $\times 13-22 \mu \mathrm{m}$. Pseudoventouses 30-57 $\times 22-44 \mu \mathrm{m}$. Ventouse ventrale $35-66 \times 35-66 \mu \mathrm{m}$. Organe tribocytique $63-150 \times 62$ $180 \mu \mathrm{m}$ (fig. $1 d, 2 d$ ).

- Se rencontre chez les Percidae, Percopsidae et Salmonidae.

- Localisation variée: reins (Coregonus), paroi de la vessie gazeuse (Perca), cœur (Acerina), rarement dans d'autres organes. Cf. Hughes (1928) ; Razmaškin (1963, 1966, 1974, 1976).

- Région holarctique.

Entre les métacercaires de 1 . platycephalus et de 1 . variegatus, il existe en outre des différences dans le spectre des Poissons, deuxièmes hôtes intermédiaires. $I$. variegatus a été trouvé surtout chez Perca fluviatilis et chez un petit nombre d'autres espèces tandis que 1 . platycephalus se rencontre dans un grand nombre de Poissons. Il est aussi utile de tenir compte de la localisation préférentielle dans tous ces hôtes. Bien entendu, ces différences n'ont qu'une importance secondaire dans les critères diagnostiques par rapport à ceux basés sur la morphologie de la métacercaire, mais ils peuvent servir de caractères d'appoint pour séparer les larves lors des examens dans la pratique.

Les expériences d'Odening, Mattheis et Bockhardt (1970) et d'Odening et Bockhardt (1971) ainsi que celles d'autres chercheurs (Van Haitsma avec «Cotylurus michi- 
ganensis », Razmaškin, Šigin) ont démontré que des métacercaires du type Tetracotyle percaefluviatilis données avec la nourriture à des Oiseaux, ont toujours provoqué une infestation de la partie postérieure de l'intestin grêle (c'est-à-dire par des adultes de I. variegatus), tandis que les métacercaires du type Tetracotyle ovata, ont provoqué une infestation au niveau du cloaque et de la Bursa Fabricii (c'est-à-dire par des adultes de I. platycephalus).

Dubois (1978) fait remarquer que la mise en synonymie de $I$. variegatus et I. platycephalus pourrait s'appuyer sur la règle écologique selon laquelle deux espèces étroitement apparentées ne peuvent coexister dans le même biotope si leurs exigences écologiques sont identiques.

La localisation différente des deux espèces dans l'hôte définitif nous paraît une réponse à cette règle. Dubois (1978) essaye d'expliquer cette localisation différente par des phénomènes d'immunité (le déplacement vers la région du cloaque résulterait d'une « fuite»). Cette interprétation n'est pas confirmée par les expériences réalisées sur le cycle de ces deux espèces. Les résultats d'Odening, Mattheis et Bockhardt (1970), d'Odening et Bockhardt (1971) et d'autres auteurs, démontrent que dès le début de l'infestation $\left(1,3,5,6,7,8-9,9,11,12,14^{\circ}\right.$ jours), I. p!atycephalus est un parasite de la région cloacale tandis que $I$. variegatus $\left(2,3,5,6,9,13\right.$ et $16^{\circ}$ jours), est localisé dans le tiers postérieur de l'intestin grêle.

Bien entendu les résultats sont différents lorsque les tentatives d'infestation ne sont pas précédées par une séparation de métacercaires selon leur morphologie. Dans la nature, il arrive assez souvent que les métacercaires de deux espèces d'Ichthyocotylurus coexistent dans un poisson. Si ce poisson est donné comme nourriture, l'hôte définitif présentera une infestation mixte qui se traduit par la présence de Trématodes dans la partie postérieure de l'intestin grêle et dans le cloaque. Une telle expérience ne peut être considérée comme la preuve de l'identité des deux espèces comme Dubois (1978) l'a pensé. Dubois signale les résultats de Swennen aux Pays-Bas qui a donné à deux Larus argentatus des métacercaires d'Acerina cernua. Or Odening et Bockhardt (1971) et d'autres auteurs (par exemple Razmaškin, 1966) ont signalé que les métacercaires des deux Ichthyocotylurus peuvent se trouver chez Acerina cernua et que leur localisation dans ce poisson est superposée.

I. platycephalus préfère la région abdominale (le plus souvent le péricarde) et I. variegatus préfère la paroi de la vessie gazeuse et le péritoine. Swennen a donné des métacercaires du péricarde à un Larus argentatus $\mathrm{A}$ et celle du mésentère à un L. argentatus B. D'après notre interprétation, il a retrouvé : chez le Goéland A 105 I. platycephalus et 262 I. variegatus; chez le Goéland B 145 I. platycephalus et 681 I. variegatus.

L'histoire, riche en péripéties, des deux « grandes » espèces d'Ichthyocotylurus est exposée en détail dans les travaux qu'Odening, Mattheis et Bockhardt (1970) et Odening et Bockhardt (1971) ont consacrés à ces Trématodes. Ils y expliquent aussi le problème compliqué de Tetracotyle ovata von Linstow, 1877. T. ovata est la métacercaire de 1. platycephalus, ceci est confirmé par l'analyse morphologique et par les résultats des infestations expérimentales. Cependant, il en est tout autrement pour une métacercaire 
que Braun (1894 $a, b)$ d'après les frères Ehrhardt a attribuée à $T$. ovata. A ce sujet Dubois (1978) écrit : «Il faut rappeler ici que Szidat (1929, pp. 741-742, fig. 29-31), pour n'avoir pas eu sous les yeux les originaux de Creplin, identifia par erreur des exemplaires de Cotylurus pileatus (Rud.), obtenus par les frères Ehrhardt à partir du Tetracotyle ovata, avec C. variegatus (Creplin). Cette erreur a été rectifiée dans la « Monographie des Strigeida ( 1938 , pp. 136-137) et dans la «Synopsis des Strigeidae et des Diplostomatidae » (1968, p. 219). »

Ainsi Dubois estime (comme il l'avait fait en 1938 et 1968, et aussi comme l'avaient pensé Hughes (1928), Szidat (1929) et d'autres encore) que les frères Ehrhardt ont réalisé leurs expériences avec le véritable $T$. ovata (ce qui n'est manifestement pas le cas). L'erreur, lourde de conséquences, est que Braun (1894 $a, b)$ a identifié les métacercaires des expériences des frères Ehrhardt à $T$. ovata.

Les adultes obtenus expérimentalement par les frères Ehrhardt ont été attribués par Braun (1894 $a, b)$ à «Holostomum variegatum» (nom employé à cette époque pour l'ensemble $I$. variegatus, $I$. platycephalus) (5), ce qui a amené Hughes (1928) à changer le nom de $T$. ovata en « $T$. variegata .

Szidat (1929) a attribué ces exemplaires des frères Ehrhardt à son Cotylurus variegatus, espèce qu'il a isolée de $C$. platycephalus. Mais le $C$. variegatus au sens de Szidat se réfère vraisemblablement en partie à Ichthyocotylurus variegatus, en partie à I. pileatus (espèce dont on n'a pas tenu compte à cette époque).

On ne peut reprocher à Szidat de ne pas avoir reconnu le caractère hétérogène de son Cotylurus variegatus car il faudrait adresser le même reproche à Dubois (1938, 1968 ) qui a (sans justification) mis en synonymie $C$. variegatus et $C$. pileatus et qui a nommé $T$. ovata, « $T$. pileata .

Il n'était pas nécessaire de réétudier le matériel original (qui d'après Szidat [1929] avait disparu) pour éviter la confusion entre $J$. variegatus et $I$. pileatus. Il suffisait d'étudier attentivement le travail de Creplin de 1825. Il semble que cela n'a été réalisé que par Odening, Mattheis et Bockhardt (1970) et par Odening et Bockhardt (1971) ce qui a permis d'éclaircir cette confusion. Cela n'enlève pas l'intérêt de la redécouverte et de la redescription par Dubois (1978), des matériels originaux de Creplin, ce qui comble une lacune dans la connaissance des deux espèces. Mais il ne nous est pas possible de le suivre dans ses conclusions qui démontrent les limites d'une systématique des Trématodes exclusivement basée sur la morphologie des adultes.

D'après la description originale de von Linstow (1877) la métacercaire nommée Tetracotyle ovata ne correspond ni à $I$. variegatus ni à $I$. pileatus (6).

(5) Dubois (1978) en mettant en synonymie $I$, variegatus avec $I$. platycephalus se réclame du droit du premier auteur réalisant une révision (C.I.N.Z., art. 24). Mais Brandes (1890) a déjà fait un synonyme, aussi injustifié, de ces deux espèces mais en sens inverse puisqu'il considérait $I$. platycephalus comme synonyme de 1 . variegatus.

(6) Razmaškin $(1963,1966)$ a prouvé expérimentalement que Tetracotyle diminuta Hughes, 1928 correspond à I. pileatus. Si Sudarikov (in Skrjabin, 1974) avait tenu compte de ces travaux de Razmaškin qui donnent des descriptions détaillées, des mesures précises et une bonne illustration de ces Trématodes, il n'aurait pas mis en doute ses résultats sur $T$. diminuta. 
Il est particulièrement regrettable que l'assimilation erronée de cette métacercaire se retrouve dans toute la littérature spécialisée d'Helminthologie et d'Ichthyoparasitologie et que la confusion qu'elle a entraînée continue à persister (par ex. Sudarikov in Skrjabin, 1959, 1971 ; Yamaguti, 1971).

A chacune des quatre métacercaires d'Ichthyocotylurus ainsi différenciées, correspond une forme adulte. Ces quatre espèces d'Ichthyocotylurus Odening, 1969 ont déjà été distinguées par Dujardin en 1845, Diesing en 1850, von Linstow en 1878 (Holostomum platycephalum, $H$. variegatum, $H$. erraticum, $H$. pileatum).

Brandes (1890) a considéré Holostomum platycephalum comme synonyme de H. variegatum.

Plus tard, pendant des dizaines d'années, les espèces 1 . pileatus et $I$. variegatus ont été passées sous silence. Depuis, en ne tenant pas suffisamment compte de la littérature originale :

- Szidat (1929) a amené sous le nom de Cotylurus variegatus une confusion entre $C$. variegatus et $C$. pileatus ;

- Dubois $(1938,1968)$ a présumé C. variegatus synonyme de C. pileatus et des caractéristiques de $C$. variegatus ont été incluses dans la diagnose de $C$. platycephalus ;

- Šigin (1961, 1966) et Razmaškin (1963, 1966) ont nommé C. variegatus «C. platycephalus» et $C$. platycephalus « $C$. communis» (nom de la forme ou sousespèce nord-américaine de platycephalus) (7).

Aperçu des caractéristiques des 4 espèces d'Ichthyocotylurus discutées ci-dessus (pour une clé de détermination, voir Razmaškin, 1966).

\section{Icbtbyocotylurus platycepbalus (Creplin, 1825)}

Synonymes :

Amphistoma platycephalum Creplin, 1825.

Holostomum cucullus Thoss, 1897.

Cotylurus communis (Hughes, 1928) La Rue, 1932.

Longueur: jusqu'à $12 \mathrm{~mm}$. Localisé dans la région du cloaque et dans la bourse de Fabricius (rarement dans le rectum). Parasite de Laro-Limicolae (Lari, Alcae), Gaviiformes et Podicipediformes, plus rarement de Pelecaniformes et Acciptriformes, exceptionnellement chez les Anseriformes. Région holarctique.

\section{Icbtbyocotylurus variegatus (Creplin, 1825).}

Synonymes :

Amphistoma variegatum Creplin, 1825.

(7) Razmaškin $(1974,1976)$ a employé les noms exacts d'espèces conformes aux indications originales de Creplin (1825). 
? Cotylurus strictus Endrigkeit, 1940.

Cotylurus cumulitestis Dubois, 1962.

Longueur: jusqu'à $13 \mathrm{~mm}$. Localisé dans le tiers postérieur de l'intestin grêle (rarement dans le rectum). Parasite surtout de Lari, mais aussi chez Alcae et Podicipedidae, exceptionnellement chez des Anseriformes. Région paléarctique.

\section{Icbtbjocotylurus erraticus (Rudolphi, 1809).}

Synonymes :

Amphistoma erraticum Rudolphi, 1809.

Strigea aquavis Guberlet, 1922.

Longueur : jusqu'à 4,5 mm. Localisé dans l'intestin grêle. Parasite des Lari, Gaviiformes et podicipediformes. Région holarctique.

Icbthyocotylurus pileatus (Rudolphi, 1802).

Synonymes :

Festucaria pileata Rudolphi, 1802.

Cotylurus medius Dubois et Rausch, 1950.

Longueur: jusqu'à 4,5 mm. Localisé dans l'intestin grêle. Parasite de Lari, plus rarement de Podicipedidae. Région holarctique.

\section{Conclusions}

Les conclusions de Dubois (1978) sur l'identité d'Ichthyocotylurus variegatus et I. platycephalus ne sont pas justifiées.

Creplin (1825), Dujardin (1845), Diesing (1850) et v. Linstow (1878) séparaient explicitement les adultes des deux espèces. A cette époque, la notion d'une localisation différente jouait un rôle important dans les caractéristiques des adultes, et l'on admettait que les métacercaires appartenaient à des espèces différentes.

La réalisation expérimentale du cycle évolutif complet des deux espèces a récemment confirmé cette conception. Les métacercaires de $I$. variegatus et $I$. platycephalus sont nettement séparées par leur morphologie. Leurs caractères distinctifs ne se croisent pas avec ceux de l'autre espèce. Expérimentalement et de façon constante les métacercaires de $I$. variegatus donnent chez l'Oiseau, hôte définitif, des adultes dans le tiers postérieur de l'intestin grêle, tandis que ceux de $I$. platycephalus donnent des adultes dans la région du cloaque. Il y a donc corrélation parfaite entre la morphologie des métacercaires et la localisation des adultes. 
La distinction entre les adultes des deux espèces est actuellement encore peu satisfaisante. Les cercaires, d'après les premières descriptions, paraissent présenter des différences morphologiques; une étude approfondie de leur chétotaxie serait donc utile.

\section{Bibliographie}

Brandes (G.), 1890 : Die Familie der Holostomiden. Zool. Jb., Abt. Syst., Okol. Geogr., 5, 549-604. Braun (M.), $1894 a$ : Helminthologische Notizen. IV. Zur Entwickelungsgeschichte der Holostomiden. Zbl. Bakt., Abt. I Orig., 15, 680-682.

Braun (M.), 1894 b: Zur Entwickelungsgeschichte der Holostomiden. Nach den Untersuchungen der Herren Alfred und Oscar Ehrhardt mitgetheilt. Zool. Anz., 17, 165-167.

Claparède (E.), 1858: Uber die Kalkkörperchen der Trematoden und die Gattung Tetracotyle. Z. wiss. Zool., 9, 99-105.

Creplin (F.C.H.), 1825: Observationes de Entozois. Pars I, x 86 p., Gryphiswaldiae.

Diesing (K.M.), 1850: Systema Helminthum 1, xvI +679 p., Wien.

Diesing (K.M.), 1858 : Revision der Myzhelminthen. Abtheilung: Trematoden. SB Akad. Wiss. Wien, math.-naturw. Cl., 32, 307-390.

Dollfus (R.Ph.), 1968: Les trématodes de l'histoire naturelle des helminthes de Félix Dujardin (1845). Mem. Mus. Nat. Hist. Nat. n.s., Ser. A, Zool., 54, 119-196.

Dubois (G.), 1938: Monographie des Strigeida (Trematoda). Mem. Soc. Neuchâtel. Sci. Nat., 85, $1-535$.

Dubois (G.), 1968 : Synopsis des Strigeidae et des Diplostomatidae (Trematoda). Mem. Soc. Neuchâtel. Sci. Nat., 10, 1-258.

Dubois (G.), 1978: A propos des matériels originaux de Cotylurus platycephalus et de Cotylurus variegatus (Creplin, 1825) (Trematoda: Strigeidae). Ann. Parasitol. Hum. Comp., 53, 53-62.

Dujardin (F.), 1845: Histoire naturelle des Helminthes ou Vers intestinaux. XvI $+654+15$ p., Paris.

Hughes (R.Ch.), 1928: Studies on the trematode family Strigeidae (Holostomidae). No. XIII. Three species of Tetracotyle. Trans. Am. Micros. Soc., 47, 414-433.

Linstow (O.v.), 1877 : Enthelminthologica. Arch. Naturgesch., 43, 173-198.

Linstow (O.v.), 1878 : Compendium der Helminthologie. xxII +382 p., Hannover.

Moulinié (J. J.) 1856: De la reproduction chez les trématodes endo-parasites. Mem. Inst. Nat. Genevois (1855), 3, 7-279.

Niewiadomska (K.), 1970: On the validity of Cotylurus platycephalus (Creplin, 1825) and C. cucullus (Thoss, 1897) [C. communis (Hughes, 1928)] (Trematoda, Strigeidae). Acta Parasitol. Polon., 18, 57-70.

Niewiadomska (K.) et Kozicka (J.), 1970: Remarks on the occurrence and biology of Cotylurus erraticus (Rudolphi, 1809) (Strigeidae) from the Mazurian Lakes. Acta Parasitol. Polon., 18, 487-496.

Odening (K.) et Bockhardt (I.), 1971: Der Lebenszyklus des Trematoden Cotylurus variegatus im Spree-Havel-Seengebiet. Biol. Zbl., 90, 49-84.

Odening (K.), Mattheis (Th.) et Bockhardt (I.), 1970: Der Lebenszyklus von Cotylurus c. cucullus (Thoss) (Trematoda, Strigeida) im Raum Berlin. Zool. Jb., Abt. Syst., Ókol. Geogr., 97, 125-198.

Olson (R.E.), 1970 : The life cycle of Cotylurus erraticus (Rudolphi, 1809) Szidat, 1928 (Trematoda : Strigeidae). J. Parasitol., 56, 55-63.

Razmaškin (D.A.), 1963 : Metacerkarii roda Cotylurus (otr. Strigeidida) iz ryb pskovsko-čudskogo vodoema. IV vsesojuzn. Sovešč. po Bolezn. Ryb / Tez. Dokl., Moskva. p. 88-90.

Razmaškin (D.A.), 1966: Razvitie tetrakotile iz ryb pskovsko-čudskogo vodoema. Gidrobiol. Issledov. Inst. Zool. Bot. AN Ést. SSR, 4, 306-322. 
Razmaškin (D.A.), 1974: A Kaškovskij (V.V.), Razmaškin (D.A.) et Skripčenko (E.G.) : Bolezni i parazity ryb rybovodnych chozjajstv Sibiri i Urala. Sverdlovsk, 160 p.

Razmaškin (D.A.), 1976: A Bolezni i parazity ryb ledovitomorskoj provincii /v predelach SSSR/. Sverdlovsk, 144 p. O ličinkach trematod, parazitirujuščich u ryb vodoemov Ob'-Irtyšskogo bassejna, p. 80-104.

Šigin (A.A.), 1961: Gel'mintofauna čajkovych ptic Rybinskogo vodochranilišča. Trudy darvinsk. gos. Zapovedn., 7, 309-362.

Šigin (A.A.), 1966: Epizootologičeskoe značenie rybojadnych ptic Rybinskogo vodochranilišča i puti sniženija ich vrednoj dejatel'nosti. A : Rybojadnye pticy $\mathrm{i}$ ich značenie $\mathrm{v}$ narodnom chozjajstve, Moskva, p. 166-193.

Skrjabin (K.I.), 1959, 1971, 1974 : Trematody životnych i čeloveka / Osnovy trematodologii, 16, 24, 25, Moskva.

Szidat (L.), 1929 : Beiträge zur Kenntnis der Gattung Strigea (Abildg.), II. Spezieller Teil : Revision der Gattung Strigea nebst Beschreibung einer Anzahl neuer Gattungen und Arten. Z. Parasitenk., 1, 688-764.

Van Haitsma (J.P.), 1930: Studies on the trematode family Strigeidae (Holostomidae). No. XXI. Life-cycle and description of the cercaria of Cotylurus michiganensis (La Rue). J. Parasitol., $16,224-230$.

Yamaguti (S.), 1971: Synopsis of digenetic trematodes of vertebrates. Tokyo, Vol. 1, 1074 p. 